\title{
Coherent Vorticity Extraction in 3D Homogeneous Isotropic Turbulence: Influence of the Reynolds Number and Geometrical Statistics
}

\author{
Benjamin Kadoch ${ }^{1}$, Margarete Oliveira Domingues ${ }^{2}$, \\ Ingmar Broemstrup ${ }^{3}$, Lionel Larchevêque ${ }^{4}$, Kai Schneider ${ }^{1,5}$ and Marie Farge ${ }^{6}$ \\ ${ }^{1}$ M2P2-CNRS and Aix-Marseille Université, 38 rue F. Joliot-Curie, 13451 Marseille Cedex 20, France \\ ${ }^{2}$ LAC-CTE, Instituto Nacional de Pesquisas Espaciais, \\ Av. dos Astronautas, 1758, C.P. 515, SJC São Paulo, Brazil \\ ${ }^{3}$ CMPD, University of Maryland, College Park, MD 20742, USA \\ ${ }^{4}$ IUSTI, Aix-Marseille Université, UMR CNRS 6595, 5 rue Enrico Fermi, 13453 Marseille Cedex 13, France \\ ${ }^{5}$ CMI, Aix-Marseille Université, 39, rue F. Joliot-Curie,13453 Marseille Cedex 13, France and \\ ${ }^{6}$ LMD-IPSL-CNRS, Ecole Normale Supérieure, 24 rue Lhomond, 75231 Paris, Cedex 05, France
}

(Received on 12 December, 2008)

\begin{abstract}
The coherent vorticity extraction method (CVE) is based on the nonlinear filtering of the vorticity field projected onto an orthonormal wavelet basis made of compactly supported functions. CVE decomposes each turbulent flow realization into two orthogonal components: a coherent and an incoherent random flow. They both contribute to all scales in the inertial range, but exhibit different statistical behavior. We apply CVE to $256^{3}$ subcubes extracted from 3D homogeneous isotropic turbulent flows at different Taylor microscale Reynolds numbers $\left(R_{\lambda}=140,240\right.$ and 680), computed by a direct numerical simulation (DNS) at different resolutions $\left(N=256^{3}, 512^{3}\right.$ and $\left.2048^{3}\right)$, respectively. We compare the total, coherent and incoherent vorticity fields obtained by using CVE and show that few wavelets coefficients are sufficient to represent the coherent vortices of the flows. Geometrical statistics in term of helicity are also analyzed and the $\lambda_{2}$ criterion is applied to the filtered flow fields.
\end{abstract}

Keywords: wavelets, isotropic turbulence, coherent structures, direct numerical simulation

\section{INTRODUCTION}

It is commonly accepted that most turbulent flows exhibit well organized structures evolving in a random background [1]. Typically, these structures are well localized and excited on a wide range of scales.

As a matter of fact, the word coherent has many different definitions in the turbulence literature, so the focus of the method presented here is not on the coherent structures themselves, but on the noise: coherent structures are here by definition what remains after the denoising, while the noise is supposed to be Gaussian and decorrelated.

Therefore, we use the extraction of coherent flutuations out of turbulent flows using the so called coherent vorticity extraction (CVE) method [2-6]. This method is based on a orthogonal wavelet decomposition of the vorticity field, a subsequent thresholding of the wavelet coefficients and a reconstruction from those coefficients whose modulus is above a given threshold. Wavelet bases are well suited for this task, because they are made of self-similar functions well localized in both physical and spectral spaces leading to an efficient hierarchical representation of intermittent data, as in turbulent flows [1]. The value of the threshold is based on mathematical theorems yielding an optimal min-max estimator for the denoising of intermittent data [7, 8]. The aim for the present paper is to study the influence of the Reynolds number on coherent vortex extraction considering different Taylor microscale Reynolds numbers: 140,240 and 680. Furthermore we present geometrical statistics of CVE results. We study helicity for $R_{\lambda}=140$, and apply the $\lambda_{2}$ criterion to the filtered fields demonstrates the features of CVE.

This paper is organized as follows. In section 2 we present the dataset used and in section 3 the CVE algorithm. In section 4 we compare the filtering using the classical CVE with a modified CVE which filters directly the velocity fields. In
Section 5, the results of CVE applied to DNS data of homogeneous isotropic turbulence at different Taylor microscale Reynolds numbers are discussed and compared with the results obtained in section 4 for the classical CVE. A similar study has been performed in [9] for a different data set. In section 6, we focus on geometrical statistics. Section 7 presents final remarks of the paper and gives some perspectives for turbulence modeling.

\section{DATASET}

The data to be analyzed corresponds to homogeneous isotropic turbulence, with a stochastic forcing at large scales, kindly provided by P.K. Yeung and his group from Georgia Tech. The fields are computed at resolution $N=2^{3 J}$, where $N$ is the number of grid points and $J$ the number of octaves in each direction.

The computational box is periodic and its largest scale is $2 \pi$ in each direction, therefore the computational grid has a step size $\Delta x=\frac{2 \pi}{N^{1 / 3}}$.

We are analyzing fields which have been computed at resolutions of $N=256^{3}, 512^{3}$ and $2048^{3}$ which correspond to microscale based Reynolds numbers $R_{\lambda}=140,240$ and 680, respectively. We focus for reasons of computational complexity to subcubes of size $256^{3}$. More details about these simulations can be found in [10]. The microscale Reynolds number is defined as $R_{\lambda}=u^{\prime} \lambda / v$ where $v$ is the kinematic viscosity, the Taylor microscale $\lambda^{2}=u^{\prime 2} /\left\langle(\partial u / \partial x)^{2}\right\rangle$ and $u^{\prime 2}=\left\langle u^{2}\right\rangle$ is a velocity scale. 


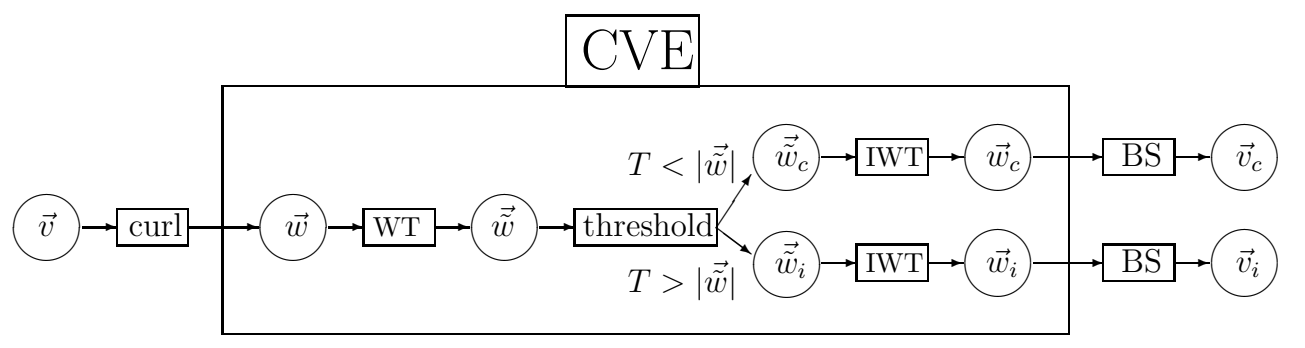

FIG. 1: Flowchart of the CVE decomposition.

\section{COHERENT VORTEX EXTRACTION}

The CVE decomposition uses an orthogonal threedimensional multiresolution analysis (MRA) of $L^{2}\left(\mathbb{R}^{3}\right)$ obtained through the tensor product of three one-dimensional MRA's of $L^{2}(\mathbb{R})$. In this context a function $f \in L^{2}\left(\mathbb{R}^{3}\right)$ can be developed into a three-dimensional wavelet basis

$$
f(\vec{x})=\sum_{\gamma \in \Gamma} \tilde{f}_{\gamma} \psi_{\gamma}(\vec{x}), \quad \gamma=\left(j, i_{x}, i_{y}, i_{z}, \mu\right)
$$

where $j$ denotes the scale, $\vec{i}=\left(i_{x}, i_{y}, i_{z}\right)$ denotes the positions, $\mu=1, \ldots, 7$ indicates the 7 wavelets and the index set $\Gamma=\{\gamma=$ $\left(j, i_{x}, i_{y}, i_{z}, \mu\right) \quad j=0, \ldots, J-1 \quad i_{x}, i_{y}, i_{z}=0, \ldots, 2^{j}-1 \quad \mu=$ $1, \ldots, 7\}$. Due to orthogonality the wavelet coefficients are given by $\widetilde{f}_{\gamma}=\left\langle f, \psi_{\gamma}\right\rangle$ where $\langle\cdot, \cdot\rangle$ denotes the $L^{2}$ inner product. For more details on this construction and on wavelets we refer the reader to the textbooks, e.g. [11] and also to the review article [1].

In the following applications, the Coifman 12 motherwavelet is chosen. This wavelet is almost symmetric and it has a compact support. Another point is that this wavelet has $M=4$ vanishing moments, and therefore the corresponding quadratic mirror filter has a length of $3 M=12$ [11].

We apply the extraction algorithm to a $3 \mathrm{D}$ vector valued vorticity field $\vec{\omega}=\nabla \times \vec{v}$, where $\vec{v}=\vec{v}(x, y, z)$ is the velocity field. The three components of $\vec{\omega}$ are developed into an orthonormal wavelet series, from the largest scale $l_{\max }=2^{0}$ to the smallest scale $l_{\text {min }}=2^{-J+1}$.

The vorticity field is decomposed into coherent vorticity $\vec{\omega}_{c}=\vec{\omega}_{c}(x, y, z)$ and incoherent vorticity $\vec{\omega}_{i}=\vec{\omega}_{i}(x, y, z)$ by projecting its three components onto an orthonormal wavelet basis and applying nonlinear thresholding to the wavelet coefficients. The choice of the threshold is based on theorems $[7,8]$ proving optimality of the wavelet representation for denoising signals - optimality in the sense that wavelet-based estimators minimize the maximum $L^{2}$-error for functions with inhomogeneous regularity in the presence of Gaussian white noise. We have chosen the variance of the total vorticity instead of the variance of the noise, which gives the threshold $T=\left(\frac{4}{3} Z \log N\right)^{\frac{1}{2}}$, where $Z=\frac{1}{2}\langle\vec{\omega}, \vec{\omega}\rangle$ is the total enstrophy (which is half the variance) and $N^{3}$ is the resolution. Notice that this threshold does not require any adjustable parameters.

In summary, first we compute the modulus of the wavelet coefficients:

$$
|\overrightarrow{\tilde{\omega}}|=\left(\sum_{n=1}^{3}\left[\tilde{\omega}_{\gamma}\right]_{n}^{2}\right)^{\frac{1}{2}}
$$

Then, the coherent vorticity is reconstructed from the wavelet coefficients whose modulus is larger than the threshold $T$, while the incoherent vorticity is computed by the difference with the total field. The two fields thus obtained, $\vec{\omega}_{c}$ and $\vec{\omega}_{i}$, are orthogonal, which ensures the decomposition of the total enstrophy into $Z=Z_{c}+Z_{i}$.

The fast wavelet transform we use, requires a total number of operations of $O(N)$, where $\mathrm{N}$ is the resolution.

The CVE decomposition algorithm consists of three fast wavelet transforms (WT) for each vorticity component, a thresholding of the wavelet coefficients and three inverse fast wavelet transforms (IWT), one for each component of $\overrightarrow{\tilde{\omega}}_{c}$, i.e., all coefficients with $|\overrightarrow{\tilde{\omega}}|$ greater than the threshold, form the coherent vorticity $\left(\vec{\omega}_{c}\right)$. The incoherent vorticity $\vec{\omega}_{i}$ components could be in principle computed using the inverse wavelet transform from the weak coefficients. In order to simplify computations we performed the difference between total and coherent vorticity which yields the same result. A flowchart of the CVE algorithm is depicted in Fig. 1. The induced coherent and incoherent velocity fields are computed using Biot-Savart's law (BS), $\vec{v}=\nabla \times\left(\nabla^{-2} \vec{\omega}\right)$, from the coherent and incoherent vorticity fields, respectively using the fast Fourier transform. As we privilege a good compression rate rather than a perfectly denoised contribution we apply the algorithm using one iteration, that is to say, we apply the filtering with a threshold computed from the incoherent part [12].

\section{COMPARISON BETWEEN FILTERING VORTICITY AND FILTERING VELOCITY}

In the previous section we presented the classical CVE where we applied the wavelet filtering to the vorticity field and subsequently reconstructed the corresponding velocity fields. Here we apply the wavelet filtering to the velocity field to get the coherent and incoherent contributions and compare it to the filtering of vorticity. Then, we compute the corresponding coherent and incoherent vorticity fields. Due to the nonlinear thresholding employed in the wavelet filtering both procedures do not yield the same result. In Table I we can observe that the compression is slightly better in the modified CVE, i.e., $1.68 \%$ instead of $3.59 \%$. This happens because the velocity field is smoother than the vorticity field. On the other hand the vorticity field computed from the curl of this velocity field is less accurate than the one obtained using CVE filtering directly applied to the vorticity field.

The 1D cuts of vorticity, shown in Fig. 2, confirm that 


\begin{tabular}{lccc|ccc}
\hline \hline & \multicolumn{6}{c}{ CVE Filtering } \\
\cline { 2 - 7 } & \multicolumn{3}{c}{ based on vorticity } & \multicolumn{3}{c}{ based on velocity } \\
\cline { 2 - 7 } & total & coherent & incoherent & total & coherent & incoherent \\
\hline \% of coeff. & 100.00 & 3.59 & 96.41 & 100 & 1.68 & 98.32 \\
\hline Energy & 2.96 & 2.95 & 0.01 & 2.96 & 2.94 & 0.02 \\
\% of Energy & 100 & 99.70 & 0.30 & 100 & 99.50 & 0.50 \\
\hline Enstrophy & 212.80 & 196.93 & 15.87 & 212.80 & 187.33 & 41.65 \\
\% of Enstrophy & 100 & 92.54 & 7.46 & 100 & 88.03 & 19.57 \\
\hline \hline
\end{tabular}

TABLE I: Comparison of filtering CVE based on a decomposition of the flow field, corresponding to a Reynolds number $R_{\lambda}=140$, using Coifman 12 wavelets and Donoho's threshold $T=1.52$ for the velocity field and $T=29.87$ for the vorticity field.

the coherent part obtained with CVE approximates well the total vorticity. However, some peaks are over estimated. In the case when the modified CVE is used, the coherent part approximates less well the total vorticity and the over estimation of the peak is worse.

(a) CVE

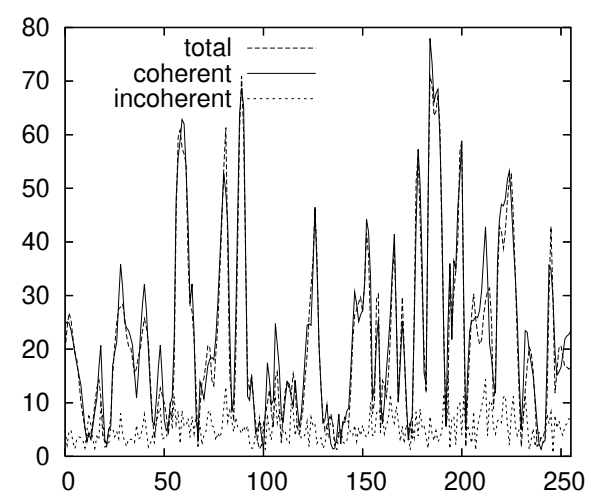

(b) modified CVE

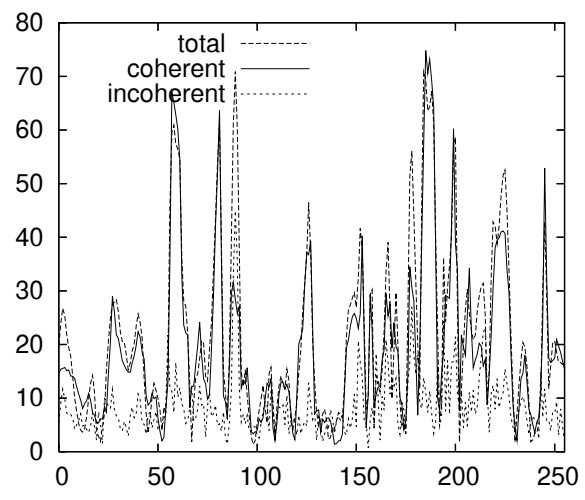

FIG. 2: One dimensional cuts in the $x$-direction, for $y=z=32 \Delta x$ with $\Delta x=\frac{2 \pi}{256}$ for the modulus of vorticity $|\vec{\omega}|$ for total field, coherent and incoherent fields at $R_{\lambda}=140$.

This explains why slightly less energy $(92.5 \%)$ is retained in the modified CVE compared to the energy retained using the vorticity based CVE (99.7\%) as illustrated in Table I.

The PDFs and spectra of the velocity on vorticity fields that were obtained with the modified CVE filtering are shown in Fig. 3 (right). The velocity and vorticity PDFs and spec- tra for the fields and the coherent contributions are similar to the ones presented in Fig. 3 (left) and that were obtained by the vorticity based CVE filtering. However the shapes of the PDFs of the incoherent fields differ for the two CVE methods. Indeed, in the case of the vorticity based CVE the PDFs of the incoherent velocity and vorticity fields are Gaussian and exponential decay, respectively, while in the case of the modified velocity based CVE the PDFs are exponential for velocity and non exponential for vorticity. Moreover, the slopes of the spectra computed from incoherent part for both methods are different.

\section{Vorticity based CVE} (a) PDF of $\vec{\omega}$

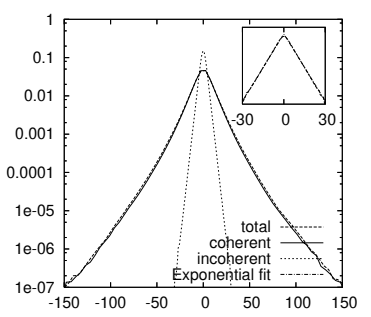

(b) spectrum of $\vec{\omega}$

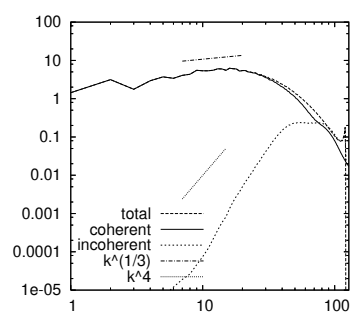

(c) PDF of $\vec{v}$

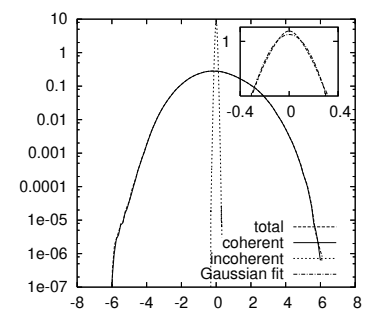

(d) spectrum of $\vec{v}$

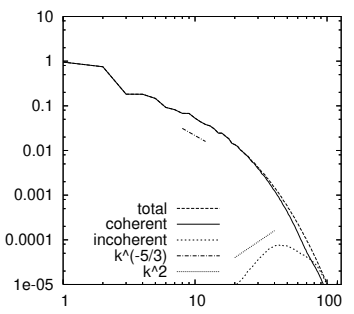

Velocity based CVE modified
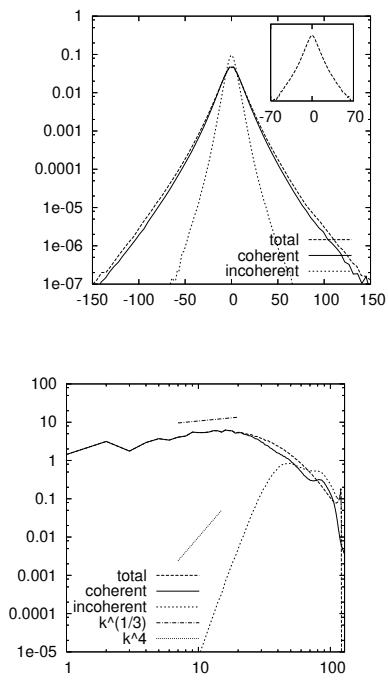

FIG. 3: PDF of the vorticity (a), velocity (c) (inset: PDF of the incoherent fields) and enstrophy (b) and energy (d) spectra for the total, coherent and incoherent fields, for the vorticity based CVE (left) and the modified velocity based CVE (right), for $R_{\lambda}=140$.

In the following we discuss the results obtained with CVE based on the filtering of the vorticity fields and applied to the vorticity at resolution $N=256^{3}$ and $R_{\lambda}=140$.

In Table I (left part for CVE based on vorticity) the statistical properties are summarized. We observe that the coherent 
part of the vorticity corresponds to $3.6 \%$ of the wavelet coefficients, which maintain $92.5 \%$ of the total enstrophy and $99.7 \%$ of the total energy.

A visualization of the modulus of vorticity and their coherent and incoherent contributions are shown in Figure 4. We observe that almost all structures are preserved in the coherent part and there are no organized structures left in the incoherent part. Indeed the total and coherent part of the vorticity fields present vortex tubes and long filaments of vorticity. The incoherent part is noise-like.

In Figure 3 (left a), vorticity PDFs are plotted for the total, coherent and incoherent parts. We find that the PDFs of the total and coherent fields almost coincide, exhibiting a stretched exponential behavior and a vorticity flatness about 8 . The incoherent part shows a strongly reduced variance and the vorticity PDF has exponential tails (with flatness 4.92).

In Figure 3 (left b, d), the energy and the enstrophy spectra for the total, coherent and incoherent parts of the fields are illustrated. We observe that in the inertial range the coherent part presents similar energy and enstrophy spectra compared to the spectra of the total field, whereas they differ only in the dissipative range for $k \geq 30$.

\section{RESULTS FOR OTHER REYNOLDS NUMBERS}

In the following we study the influence of the Reynolds number. We apply the CVE to vorticity fields at $R_{\lambda}=240$ and 680 computed at resolution $N=512^{3}$ and $2048^{3}$, respectively. For computational reasons, we only consider subcubes of size $N=256^{3}$, and we apply the CVE to four subcubes to check if the statistics differ.

Table II summarizes the statistical properties of the vorticity for four different subcubes of size $256^{3}$ extracted from the $N=512^{3}$ cube which correspond to a Reynolds number $R_{\lambda}=240$ and for four $256^{3}$ subcubes of the $N=2048^{3}$ simulation which correspond to a Reynolds number $R_{\lambda}=680$.

For $R_{\lambda}=240$, the reconstruction of the coherent part of these fields requires only $3.6 \%$ of the wavelet coefficients and retains $\approx 92 \%$ of the enstrophy, and for $R_{\lambda}=680$ only $\approx 3.7 \%$ of the wavelet coefficients maintain about $91 \%$ of the enstrophy. Additionally, there are no significant changes in the skewness and flatness between the total field and the coherent field for the different $R_{\lambda}$. Moreover, we do not observe significant variations between the statistical properties of the four different subcubes for each $R_{\lambda}$. The total, coherent and incoherent parts of the vorticity fields are visualized in Figure 4 for $R_{\lambda}=140,240$ and 680. As observed in the $R_{\lambda}=140$ case, all structures are preserved in the coherent contribution and none remain in the incoherent contribution. However, with the increase of $R_{\lambda}$, we observe the presence of more structures in the analyzed subcubes.

In Fig. 5 the PDFs of the vorticity and the enstrophy spectra for the total, coherent and incoherent fields are plotted for $R_{\lambda}=240$ and 680 . They have the same behavior as the vorticity field for $R_{\lambda}=140$, presented in Fig. 3 (a,b).

In [9] a related work was done also for homogeneous isotropic turbulence. The CVE was used for different resolutions $256^{3}, 512^{3}, 1024^{3}$ and $2048^{3}$ corresponding to $R_{\lambda}=167$, 257,471 and 732, respectively. The main difference is that

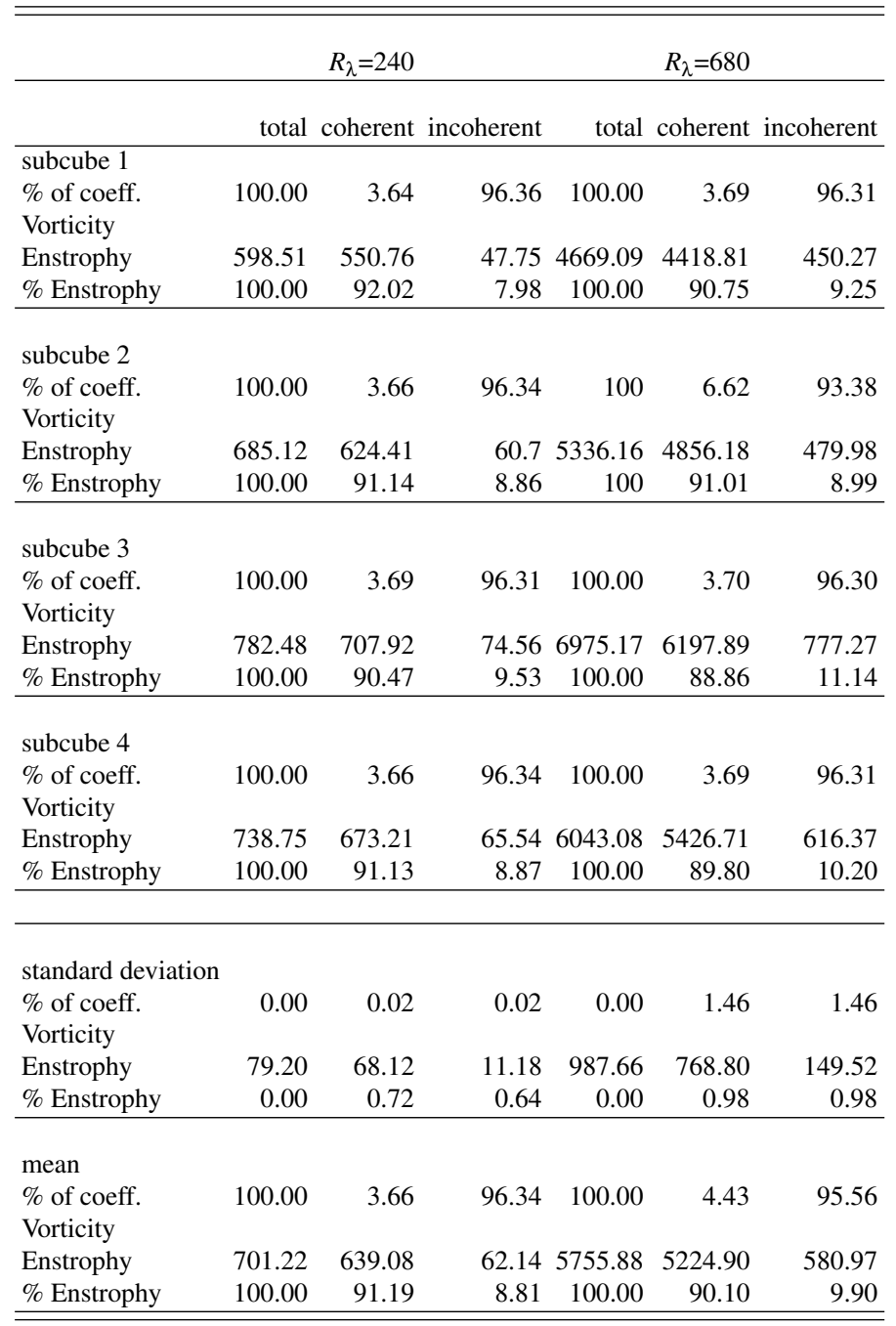

TABLE II: Statistical properties of the vorticity field for the $256^{3}$ subcubes extracted from the $512^{3}$ and $2048^{3}$ which correspond to Reynolds numbers $R_{\lambda}=240$ and $R_{\lambda}=640$, respectively. The value of Donoho's threshold used for the subcubes 1 to 4 for $R_{\lambda}=140$ are $51.18,56.39,61.55$ and 58.56, respectively. The value of Donoho's threshold used for the subcubes 1 to 4 for $R_{\lambda}=640$ are 152.23, $157.58,192.21$ and 174.20 , respectively.

the CVE was applied to the whole fields and not on subcubes, as done here. The authors find that the compression rate increases with $R_{\lambda}$. In our analysis on the subcubes, the compression rates are nearly constant, about $3.6 \%$ for the different $R_{\lambda}(140,240,680)$, so that, the Donoho threshold increases with $R_{\lambda}$. This could suggest that the compression rate is limited by the size of the box. Indeed the resolution of all cubes we analyzed is constant at $256^{3}$. Moreover, the decay of coefficients is smaller for the subcubes, but the PDFs and spectra are very similar to the ones obtained in our case. 
(a) $|\vec{\omega}|$
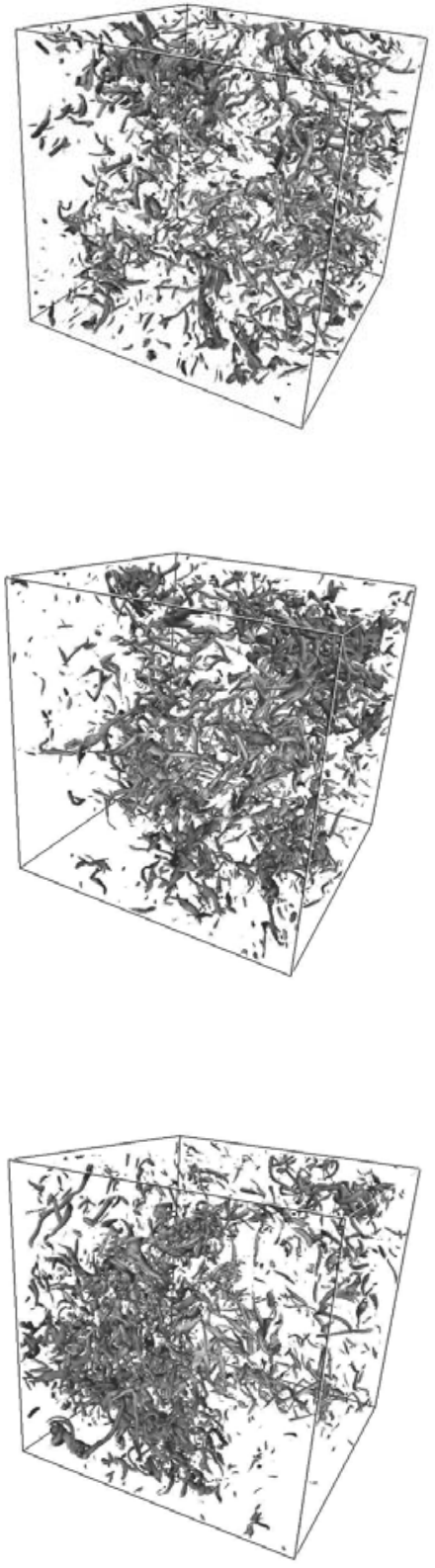

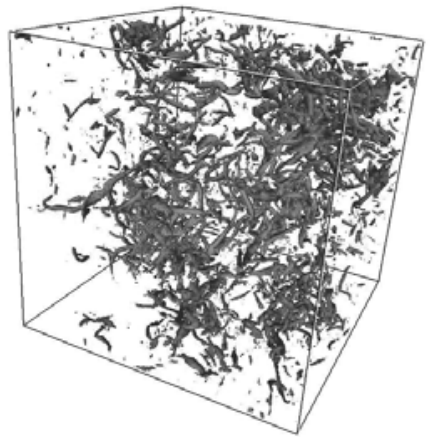

$R_{\lambda}=240$

(b) $\left|\vec{\omega}_{c}\right|$

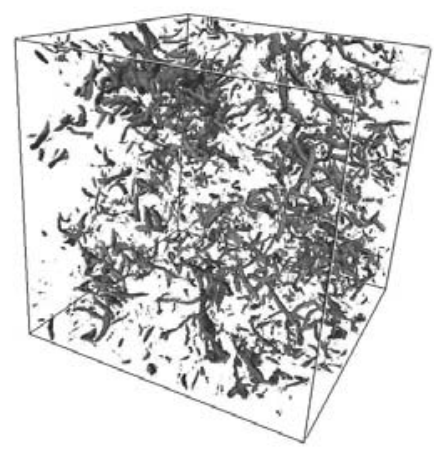

$R_{\lambda}=140$

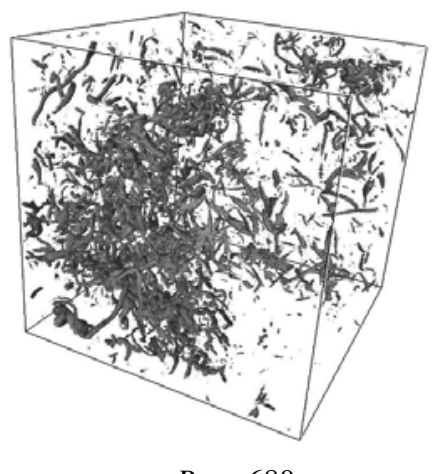

(c) $\left|\vec{\omega}_{i}\right|$
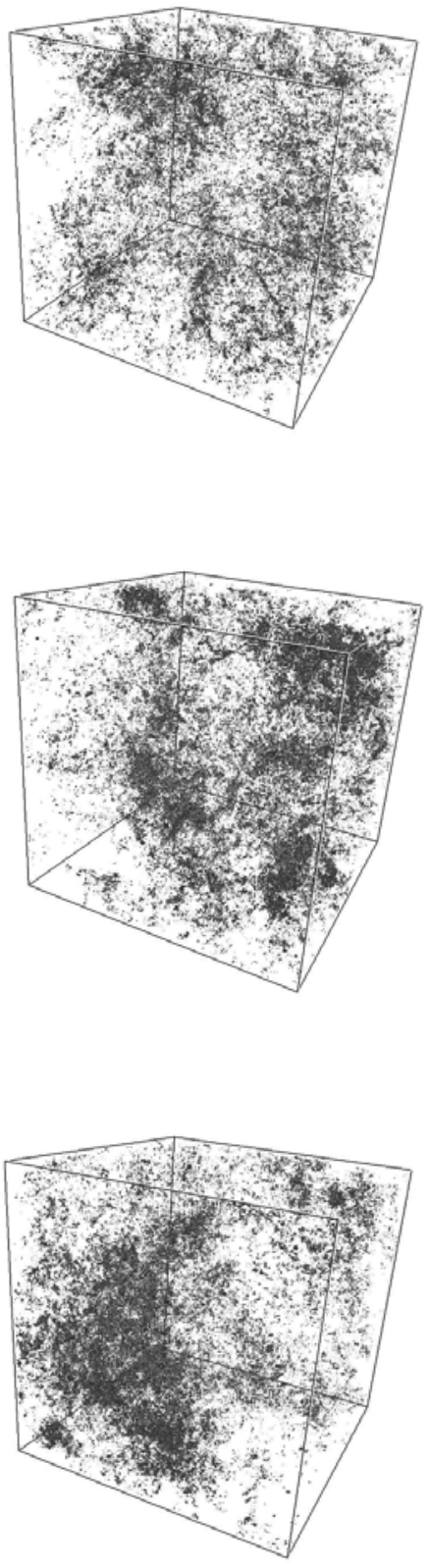

FIG. 4: Modulus of vorticity for total (a), coherent (b), and incoherent (c) parts. $R_{\lambda}=240$ and $R_{\lambda}=680$ subcubes $256^{3}$ are visualized to zoom on the structures. The values of the isosurfaces are $\sigma_{140}=56, \sigma_{240}=96$ and $\sigma_{680}=290$ for total and coherent parts and $\sigma_{140}=14, \sigma_{240}=24$ and $\sigma_{680}=75.2$ for the incoherent part.

\section{GEOMETRICAL STATISTICS}

\section{VI.1. Helicity}

To get insight into the geometrical statistics of the flow we study the helicity [13] defined by

$$
H(\vec{v}, \vec{\omega})=\vec{v} \cdot \vec{\omega} .
$$

To study the effect of CVE we split the helicity into four contributions, namely

$$
\begin{aligned}
& H_{c c}=h\left(\vec{\omega}_{c}, \vec{v}_{c}\right), \quad H_{i c}=h\left(\vec{\omega}_{i}, \vec{v}_{c}\right), \\
& H_{c i}=h\left(\vec{\omega}_{c}, \vec{v}_{i}\right), \quad H_{i i}=h\left(\vec{\omega}_{i}, \vec{v}_{i}\right),
\end{aligned}
$$

corresponding to the coherent and incoherent contributions of the velocity and vorticity components.

In [3] it was proposed to consider the local Beltramization of the flow to characterize coherent vortices which correspond to regions where the nonlinearity is depleted and which max- 

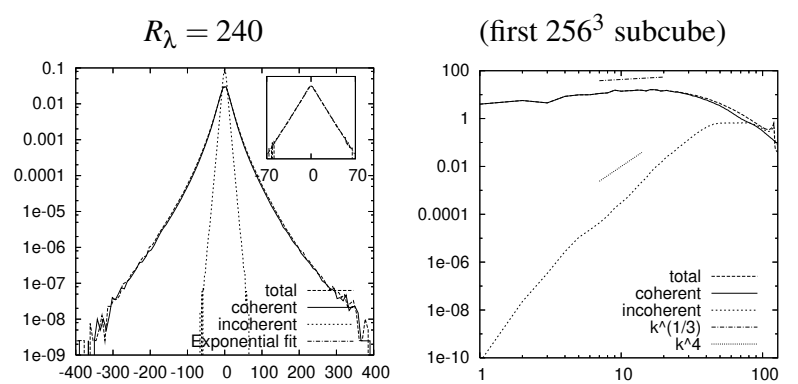

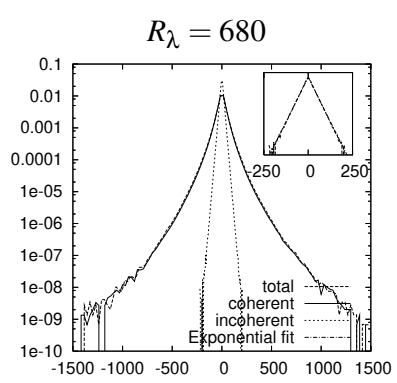

(a) PDF of $\vec{\omega}$

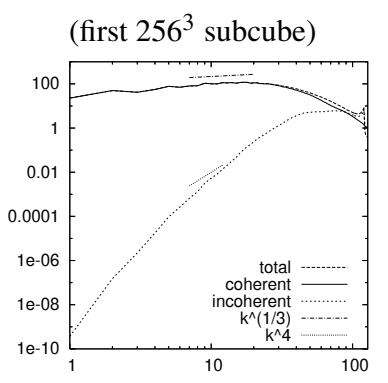

(b) Enstrophy spectrum
FIG. 5: PDF (left) of vorticity (inset: PDF of the incoherent fields) and enstrophy spectrum (right) for the total field, coherent and incoherent parts.

imize the relative helicity, defined as

$$
h(\vec{v}, \vec{\omega})=\frac{\vec{v} \cdot \vec{\omega}}{\|\vec{v}\|\|\vec{\omega}\|} .
$$

Such a characterization corresponds to a special case of nonlinearity depletion. However the latter does not prove that we have extracted all coherent vortices using the CVE decomposition. Also the relative helicity is split into four contributions, namely

$$
\begin{aligned}
& h_{c c}=h\left(\vec{\omega}_{c}, \vec{v}_{c}\right), \quad h_{i c}=h\left(\vec{\omega}_{i}, \vec{v}_{c}\right), \\
& h_{c i}=h\left(\vec{\omega}_{c}, \vec{v}_{i}\right), \quad h_{i i}=h\left(\vec{\omega}_{i}, \vec{v}_{i}\right) .
\end{aligned}
$$

In Fig. 6 we show visualizations of the relative helicity for the four components. Almost all of the structures are present in the $h_{c c}$ component. We can observe also some small structures in $h_{c i}$ and $h_{i c}$, and no more structures in $h_{i i}$. This can also be observed in the PDF of the helicity. The PDFs of the different contributions of relative helicity are plotted in Figure 7. The coherent velocity, i.e. either $h_{c c}$ or $h_{i c}$, exhibits the same PDF as the one of the total flow, with two maxima at $h= \pm 1$ for the relative helicity.

This corresponds to helical vortex tubes for which velocity and vorticity vectors are either parallel or anti-parallel, respectively. On the other hand, the PDF of the relative helicity based on the incoherent velocity and the incoherent vorticity, i.e., $h_{i i}$, is maximal at $h=0$ which suggests a tendency towards two-dimensionalization.

\section{VI.2. The $\lambda_{2}$ criterion}

The coherent nature of the structures extracted by wavelet analysis can be assessed by comparing the coherent structures educed from both the total and wavelet-filtered flow fields by means of one of the classical criteria used to define a vortex core. Among these criteria, the $\lambda_{2}$ [14] criterion is of interest since it yields an accurate eduction of the vortices, whereas the physical analysis on which it is based is not directly related to the vorticity. It therefore allows a fair analysis for the accuracy of the vorticity-based wavelet extraction.

The key idea behind the $\lambda_{2}$ criterion is to seek for regions exhibiting relative minima of pressure classically associated with vortical structures while discarding viscous and unsteady effects that are not related to the vortex physics. After some rearrangements of the incompressible Navier-Stokes equations taking into account these restrictions (see Jeong and Hussain[14] for details), the Hessian tensor of pressure $\mathcal{H}(p)$ is approximated by the opposite of the tensor $\mathrm{S}^{2}+\Omega^{2}$ where $S$ and $\Omega$, respectively, stand for the symmetric and antisymmetric part of the velocity gradient tensor. Regions of pressure minima are characterized by two positive eigenvalues for the pressure Hessian, implying that the second highest eigenvalues $\lambda_{2}$ of the tensor $S^{2}+\Omega^{2}$ is negative in such regions. Vortex core in the sense of the $\lambda_{2}$ criterion are consequently educed by the condition :

$$
\lambda_{2}^{S^{2}+\Omega^{2}}<0
$$

The original definition of the $\lambda_{2}$ criterion is generally useless for visualization of fully turbulent flows since it yields the eduction of a very large amount of the total volume. One way to alleviate this drawback is to arbitrary set a threshold lower than the original 0 -valued one. From a mathematical viewpoint, it implies that only the vortex core exhibiting the steepest pressure minima are highlighted. Physically, the cores thus educed correspond to the strongest and most coherent structures.

The value of the isosurface has been set for the present analysis to a value roughly equal to the twice of the opposite of the standard deviation of $\lambda_{2}$ over the whole computational volume. It is worth noting that the values of the standard deviation found for the total and coherent flow field are almost identical. Consequently, the same eduction threshold has been applied to both flow fields. Structures educed from the total and coherent flow field are displayed in Figs. (8) (a) and (b), which demonstrate that the original topology of the vortices is almost perfectly recovered from the coherent part of the wavelet-filtered flow field. On the contrary, if the value of the isosurface level is increased up to four times the opposite of the $\lambda_{2}$ standard deviation value over the incoherent flow field, as seen in Fig. 8 (c), structures are indeed educed but they are of small sizes, being almost isotropic in shape, and they hardly exhibit any spatial organization. It can therefore be concluded that the $\lambda_{2}$ analysis a posteriori demonstrates that the CVE effectively result in the extraction of coherent vortices in one of the most widely accepted sense.

\section{FINAL REMARKS}

CVE has been applied to 3D homogeneous isotropic turbulent flows for three different Reynolds numbers. We decomposed the flows into their coherent parts, made of vortex tubes, which interact nonlinearly, and into an incoherent 
(a) $|h|$

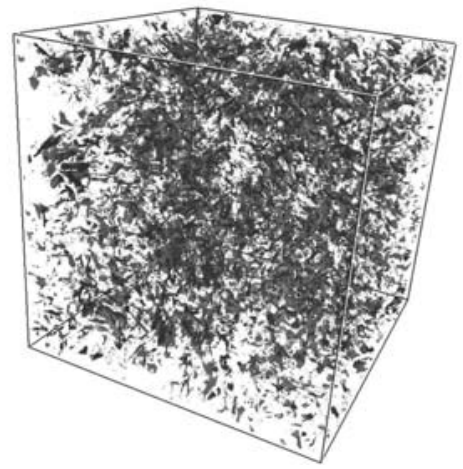

(b) $\left|h_{c c}\right|$

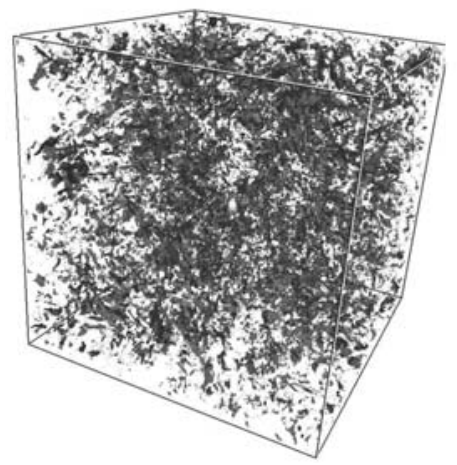

(c) $\left|h_{c i}\right|$

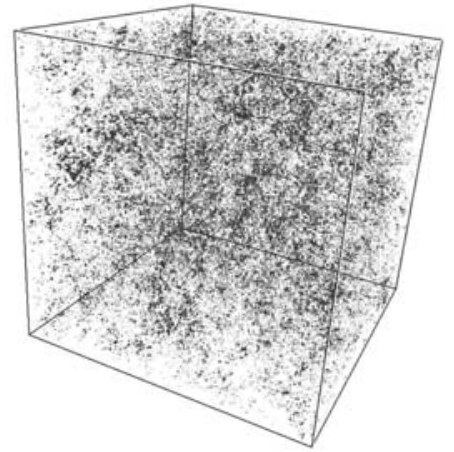

(d) $\left|h_{i c}\right|$

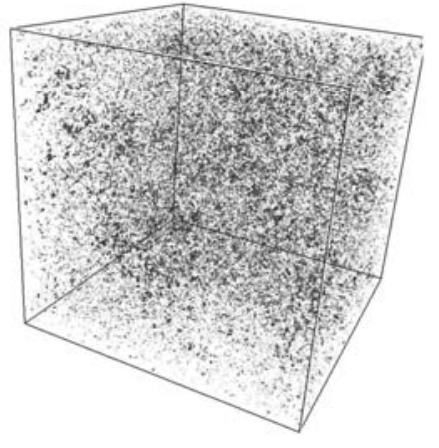

(e) $\left|h_{i i}\right|$

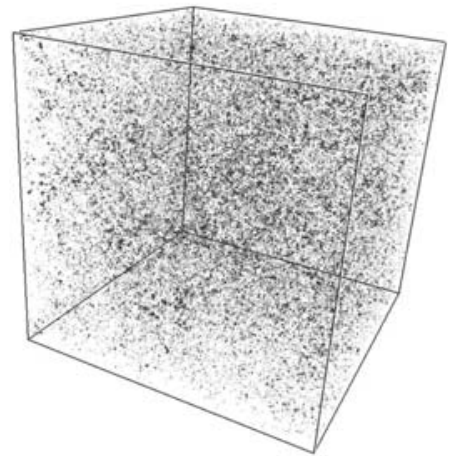

FIG. 6: Total helicity (a) and the four contributions of relative helicity for $R_{\lambda}=140$. The isosurfaces are $|\sigma|=\left|\sigma_{c c}\right|=0.97$ (b), $\left|\sigma_{c i}\right|=0.95$ (c), $\left|\sigma_{i c}\right|=0.95$ (d) and $\left|\sigma_{i i}\right|=0.94$ (e). The red color indicates positive isosurfaces and the blue color negative values.

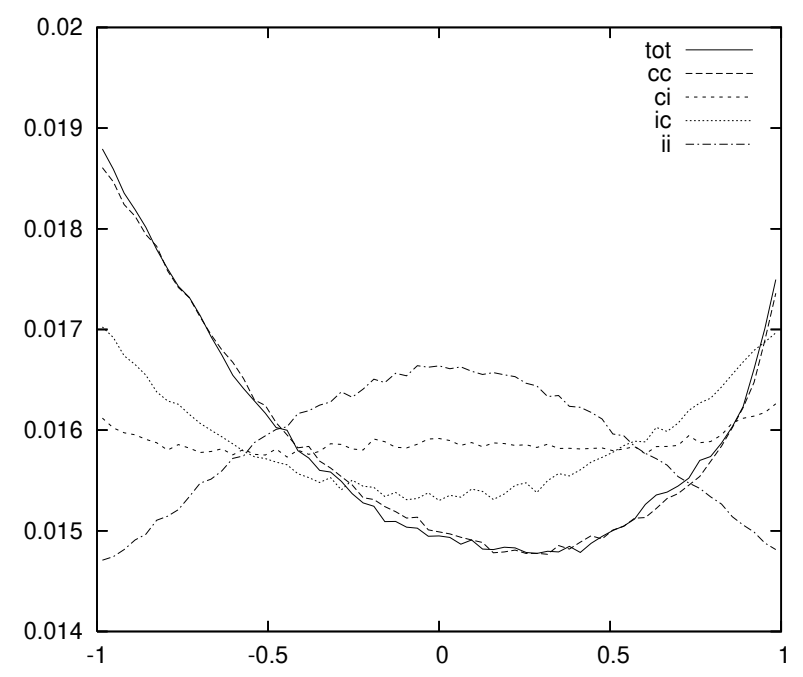

FIG. 7: PDFs of the relative helicity for the total and the four contributions.

random background flow, which is structureless and decorrelated. The above results hold for all Reynolds numbers we investigated. So we have shown that the CVE method is an efficient tool for extracting coherent vortices out of turbulent flows.

From the present results we conjecture that modeling the effect of the discarded modes on the resolved modes is easier to perform using the Coherent Vortex Simulation (CVS) approach introduced in $[4,15]$. CVS computes all degrees of freedom which contribute to the flow nonlinearity, i.e., the coherent modes, whatever their scale, while the remaining degrees of freedom, i.e., the incoherent modes, are discarded to model turbulent dissipation. The method actually combines an Eulerian projection of the solution with a Lagrangian procedure for the adaption of the computational basis: for more details we refer the reader to [15]. The next step to demonstrate the potential of CVS is to develop an adaptive wavelet solver for the 3D Navier-Stokes equations.

\section{Acknowledgments}

We are very thankful to Prof. P. K. Yeung and Dr. D. Donzis from Georgia Tech for the dataset they provided us. Visualizations in this paper were produced using VAPOR (www.vapor.ucar.edu), a product of the National Center for Atmospheric Research [16, 17]. The authors thank J. Clyne and A. Norton to introduce us to this software. This work was supported by the ANR project "M2TFP", the DFG-CNRS Research Program "LES and CVS of Complex Flows". M. O. Domingues acknowledges M2P2-CNRS, Aix-Marseille Université, LMD-IPSL-CNRS, Ecole Normale Supérieure, CNPq, FAPESP, ST2I department of CNRS and Ecole Cen- 
(a) $\lambda$

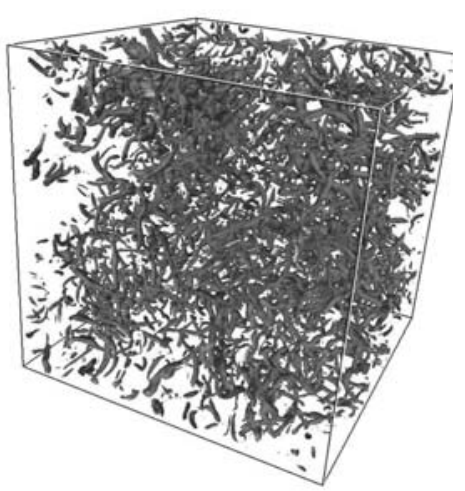

(b) $\lambda_{c}$

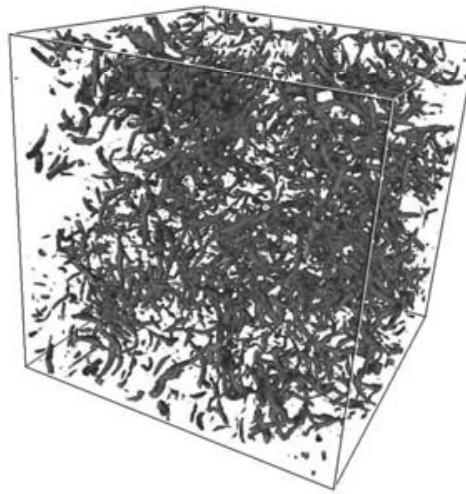

(c) $\lambda_{i}$

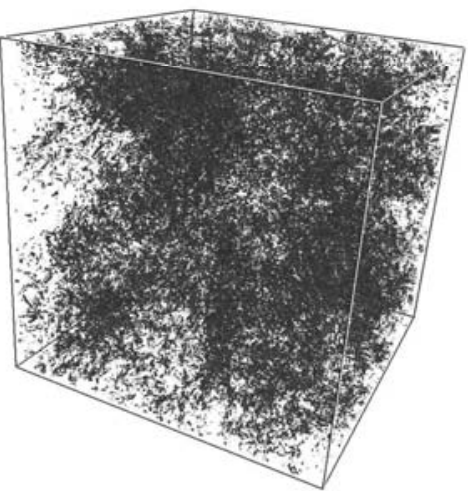

FIG. 8: Coherent structures educed by the $\lambda_{2}$ criterion from the total flow field (a), the coherent flow field (b), and the incoherent flow field (c). The isosurfaces visualized are $\sigma=0.2(\mathrm{a}), \sigma_{c}=0.2(\mathrm{~b})$, and $\sigma_{i}=0.02(\mathrm{c})$.

trale Marseille.

Related papers can be downloaded from the following web- page: 'http://wavelets.ens.fr/'

corresponding author: kadoch@13m.univ-mrs.fr
[1] M. Farge, Annu. Rev. of Fluid Mech. 24, 395 (1992).

[2] M. . Domingues, I. Broemstrup, K. Schneider, M. Farge, and B. Kadoch, ESAIM: Proc. 16, 164 (2007).

[3] M. Farge, G. Pellegrino, and K. Schneider, Phys. Rev. Lett. 87, 054501 (2001).

[4] M. Farge, K. Schneider, and N. Kevlahan, Phys. Fluids 11, 2187 (1999).

[5] M. Farge, K. Schneider, G. Pellegrino, A. A. Wray, and R. S. Rogallo, Phys. Fluids 15, 2886 (2003).

[6] K. Schneider, M. Farge, G. Pellegrino, and M. Rogers, J. Fluid Mech. 534, 39 (2005).

[7] D. Donoho, Appl. Comput. Harmon. Anal. 1, 100 (1993).

[8] D. Donoho and I. Johnstone, Biometrika 81, 425 (1994).

[9] N. Okamoto, K. Yoshimatsu, K. Schneider, M. Farge, and Y. Kaneda, Phys. Fluids 19, 115109 (2007).
[10] P. Yeung, D. Donzis, and K. Sreenivasan, Phys. Fluids 17, 081703 (2005).

[11] I. Daubechies, Ten Lectures on wavelets, vol. 61 (CBMS-NSF Conferences in Applied Mathematics, SIAM, 1992).

[12] A. Azzalini, M. Farge, and K. Schneider, Appl. Comput. Harmon. Anal. 18, 177 (2005).

[13] H. K. Moffatt, J. Fluid Mech. 150, 359 (1985).

[14] J. Jeong and F. Hussain, J. Fluid Mech. 285, 69 (1995).

[15] M. Farge and K. Schneider, Flow Turbul. Combust. 66, 393 (2001).

[16] J. Clyne, P. Mininni, A. Norton, and M. Rast, New J. Phys. 9, 301 (2007).

[17] J. Clyne and M. Rast, Proc. of Visualization and Data Analysis pp. 284-294 (2005). 